\title{
Electrical control of optically pumped electron spin in a single GaAs/AlAs quantum dot fabricated by nanohole infilling
}

\author{
S. Germanis $\odot,{ }^{1, *}$ P. Atkinson, ${ }^{1}$ R. Hostein,,${ }^{1}$ S. Suffit, ${ }^{2}$ F. Margaillan, ${ }^{1}$ V. Voliotis $\odot,{ }^{1}$ and B. Eble ${ }^{1, \dagger}$ \\ ${ }^{1}$ Institut des Nanosciences de Paris, Sorbonne Université, CNRS, 75005 Paris, France \\ ${ }^{2}$ Laboratoire Matériaux et Phénomènes quantiques, Université de Paris, CNRS, F-75013 Paris, France
}

(Received 4 March 2020; revised 16 June 2020; accepted 18 June 2020; published 6 July 2020)

\begin{abstract}
We demonstrate here electrical control of the sign of the circularly polarized emission from the negatively charged trion, going from co- to contrapolarized with respect to the circular polarization of the laser, using a GaAs/AlAs quantum dot (QD) embedded in a field effect structure. The voltage range over which the trion is negatively (contra) circularly polarized is shown to be dependent on the laser excitation energy within the $P$-shell resonance. The negative polarization never exceeds $\sim-15 \%$, in stark contrast to measurements on InAs/GaAs QDs reported by M. E. Ware et al. [Phys. Rev. Lett. 95, 177403 (2005).] in which a negative polarization reaching $-95 \%$ was observed. This result is shown to be a consequence of the low-symmetry confinement potential of these GaAs/AlAs QD, which are fabricated by partial infilling of asymmetric droplet-etched nanoholes. This low QD symmetry also leads to optical activity of the dark spin configuration of the triplet state, which we measure experimentally by photoluminescence excitation spectroscopy. A simple, semiquantitative model explaining both the optical activity of the dark spin configuration and the maximum degree of negative polarization is presented.
\end{abstract}

DOI: 10.1103/PhysRevB.102.035406

\section{INTRODUCTION}

An electron spin trapped in a semiconductor quantum dot (QD) is a robust qubit whose quantum state can be fully manipulated by laser sequences tuned to the lowest excited state, the negatively charged trion. In this case, an applied external magnetic field is imperative to lift the spin degeneracy of the trion state to create a $\Lambda$ system and initialize the spin by optical pumping techniques [1]. In contrast, the trion's excited state (hot trion), which is formed with one electron on the $S$ orbital and one electron on the $P$ orbital, gives rise to triplet states whose degeneracy is lifted by the electron-hole exchange interaction. As the total electronic spin within the negatively charged trion $X^{-}$is zero, the circular polarization of the luminescence maps the spin of the hole which recombines. When the circular polarization is negative, i.e., contrapolarized with respect to the laser excitation, an efficient flip-flop process occurs between one of the electrons and the photocreated hole spin. The spin flip flop of the electron and hole, arising before the hot trion can decay to the trion ground state, leads to initialization of the spin of the electron remaining in the QD [2]. This negative polarization effect has been used in measurements of an ensemble of $n$-doped QDs to probe the dynamics of the coupled electron-nucleus spin system in the low-magnetic-field limit [3-5].

The degree of circular polarization, defined as $P_{c}=$ $\left(I_{\sigma^{+} \sigma^{+}}-I_{\sigma^{+} \sigma^{-}}\right) /\left(I_{\sigma^{+} \sigma^{+}}+I_{\sigma^{+} \sigma^{-}}\right)$, has been shown to reach a value close to $-100 \%$ for the negatively charged trion of a

\footnotetext{
*germanis@insp.jussieu.fr

†benoit.eble@insp.jussieu.fr
}

single InAs/GaAs QD [6,7]. Here, we study the dynamics of the negative circular polarization from a strain-free, highly asymmetric single GaAs/AlAs QD grown by partial infilling of an in situ droplet-etched nanohole [8]. These QDs were embedded in a n-i-Schottky diode structure, designed to permit discrete charging of QDs with a wide voltage range where the $X^{-}$state was dominant.

We investigate here the combined effect of applied bias and excitation energy on the degree of negative circular polarization of the trion emission. While optically addressing the hot trion through $P$-shell excitation of the QD, we observe that the applied bias can be used to control the degree of negative circular polarization up to its complete disappearance. Similarly, tuning the excitation energy within the broad $P$ shell resonance can lead to suppression of either the negative or positive circular polarization. In addition, we carry out resonant excitation of the hot trion by exciting the forbidden $S-P$ transition, involving the photocreation of an electron in the conduction band $P$ orbital and a hole in the lowest-energy valence band $S$-orbital. In this configuration we observe three lines in the photoluminescence excitation (PLE) spectra of the hot trion, corresponding to the two bright triplet states and the dark spin configuration. This is in contrast to measurements of InAs/GaAs QDs, where only selective absorption of the two bright triplet states has been observed [6]. We explain the optical activity of the dark spin configuration of the hot trion by considering the anisotropic morphology of the nanohole which determines the shape of the GaAs/AlAs QDs studied in this work. This anisotropy lowers the group symmetry of the confinement potential down to $C_{s}$, which strongly modifies the optical activity of dark spin configuration excitons [9]. We develop a simple model where the electron-hole exchange 
(a)

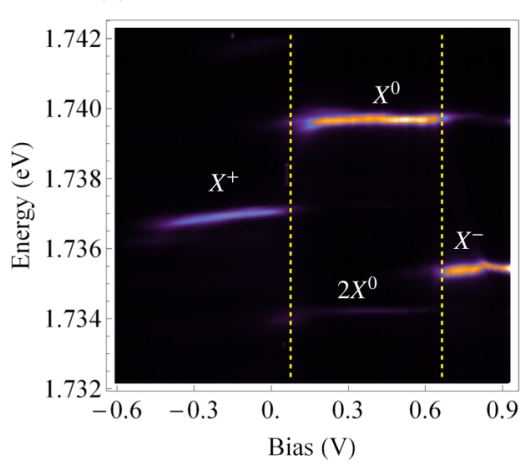

(b)

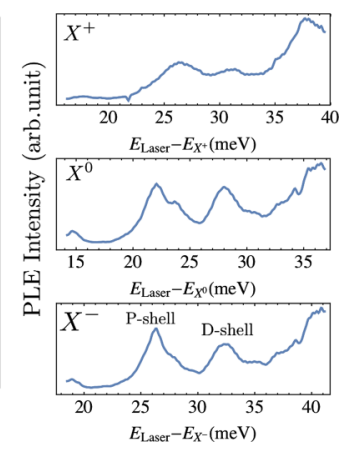

FIG. 1. (a) Map of the photoluminescence of a single QD as a function of the applied bias. The laser energy is set at $E_{\text {Laser }}=$ $1.771 \mathrm{eV}$. (b) Fast-scan PLE for the three exciton states; -0.5 , 0, and $0.7 \mathrm{~V}$ were applied to the Schottky diode to record the PLE spectra of $X^{+}, X^{0}$, and $X^{-}$, respectively.

interaction is expressed in the reduced-symmetry framework and show that it agrees well with the experimental results.

\section{EXPERIMENTS}

The sample used consisted of GaAs/AlAs QDs fabricated by nanohole infilling inside a n-i-Schottky structure with a 168-nm-wide intrinsic region. These nanoholes, created by $\mathrm{Al}$ droplet deposition followed by annealing under $\mathrm{As}_{4}$, are typically $8-10 \mathrm{~nm}$ deep and $\sim 60 \mathrm{~nm}(\sim 90 \mathrm{~nm})$ wide along [-110] ([110]). Notably, the deepest point of the hole is generally offset from the hole center due to droplet motion during etching, leading to asymmetry of the hole profile along the $[-110]$ direction [10]. The nanoholes are overgrown by a 1-nm-thick GaAs layer, but net migration towards the bottom of the hole during a short 15-s interrupt leads to the dots studied here being 2-4 $\mathrm{nm}$ high. Nanohole etching occurs after an 18-nm $\mathrm{Al}_{0.45} \mathrm{Ga}_{0.55}$ As intrinsic spacer has been grown over the $n$-doped contact, leading to dots being located nominally around $10 \mathrm{~nm}$ from the $n$ contact. However, variation in both nanohole size and infilling amount across the 2 inch wafer leads to a range in dot size and separation from the $n$ contact. In this sample, the QD density is very low (one or two QDs per square micrometer), allowing individual QDs to be easily isolated for optical spectroscopy experiments. The QD charge is controlled by applying a bias between the semitransparent Schottky gate evaporated on the top surface of the sample and the diffusive Ohmic contact. The different charged states for a typical QD emitting at $1.74 \mathrm{eV}$ are revealed in Fig. 1(a), which shows the evolution of the photoluminescence signal when sweeping the bias. Both the positive $\left(X^{+}\right)$and negative $\left(X^{-}\right)$trions are clearly identified and are well separated from the neutral exciton $\left(X^{0}\right)$ voltage domain.

Figure 1(b) shows the photoluminescence excitation (PLE) spectra for the three excitonic charge states of the same QD. The $P$-shell transition and the $D$ shell are located $\sim 26$ and $\sim 34 \mathrm{meV}$ above the lowest-energy transitions. These values agree well with the performed eight-band $\boldsymbol{k} \cdot \boldsymbol{p}$ calculations of a QD with a 50-nm radius and a 4-nm height. The electronic excitation resonances are several $\mathrm{meV}$ wide, although their

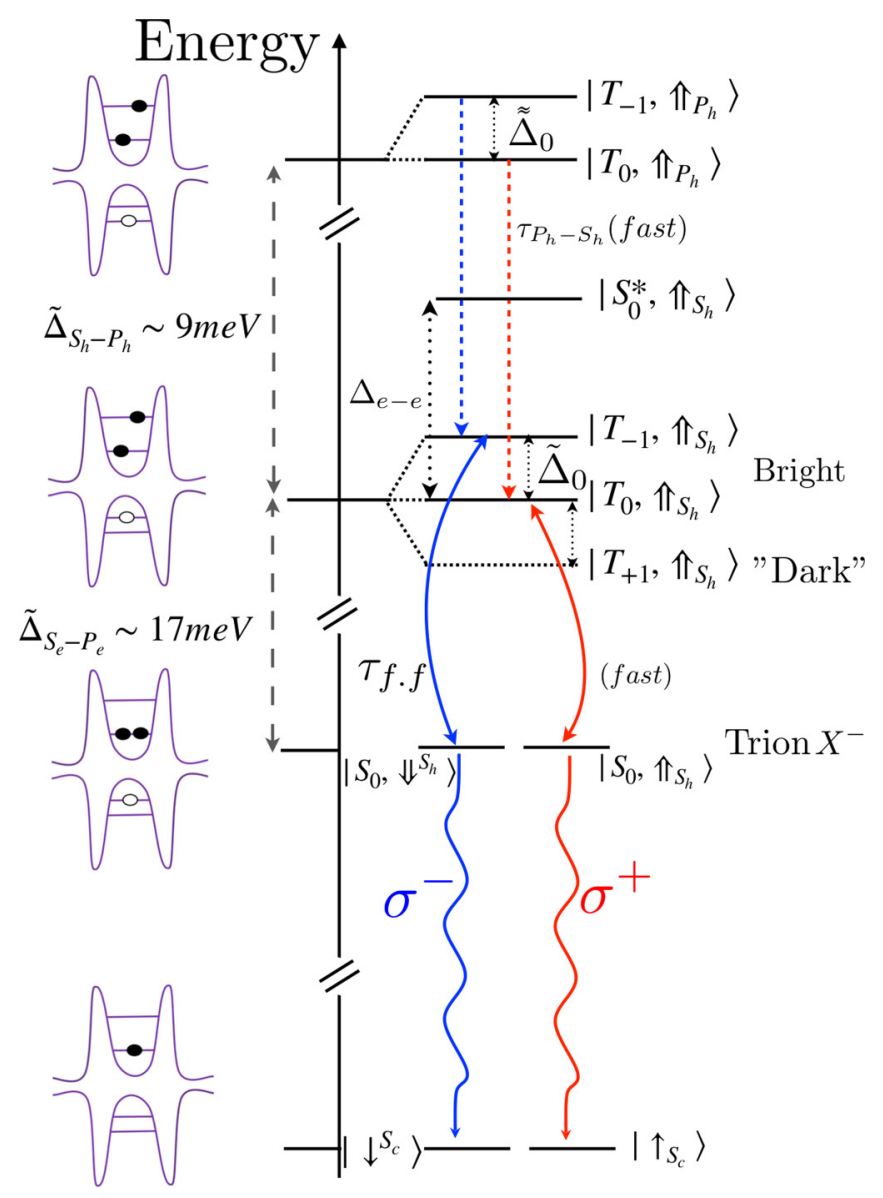

FIG. 2. Fine structure of the hot trion. The lifted degeneracy of the triplet states is fully shown when the hole occupies the $S$-orbital level, whereas only $T_{0}$ and $T_{-1}$ are represented when the hole is on the $P$-like orbital. All the states of the hot trion are twofold degenerate depending on the hole spin projection. As the QD is excited by a $\sigma^{+}$polarized laser, only the states with hole spin up are depicted. $\tilde{\Delta}_{S_{e}-P_{e}}$ and $\tilde{\Delta}_{S_{h}-P_{e}}$ are the energy differences between the orbital levels renormalized by the Coulomb interaction. The blue path gives rise to contrapolarized luminescence of the trion, whereas the red path leads to copolarized trion emission.

width decreases going from $X^{+}$to $X^{0}$ to $X^{-}$, i.e., as the bias approaches the flat-band conditions, here close to $1 \mathrm{Vt}$. This indicates that carrier tunneling contributes significantly to the broadening. However, even under strong forward bias, when the band structure is close to the flat-band condition, the resonances associated with the negative trion $X^{-}$remain broad and larger than $\sim 3 \mathrm{meV}$. We interpret this as a consequence of the very fast hole relaxation time $\left(\tau_{P_{h} \rightarrow S_{h}} \sim 1 \mathrm{ps}\right)$ from the $P$ to the $S$ orbital.

To optically address the hot trion, we first choose to excite the $P$-shell transition of the QD while polarizing the diode in the voltage range where the $X^{-}$is stable. The different configurations of charge carriers in the dot following this excitation and subsequent carrier relaxation and recombination are shown in Fig. 2 [11]. For simplicity, we consider only the lowest-energy $P$ orbital (let's say $P_{x}$ ) for the valence and the conduction band, and we discard the influence of the splitting between $P_{x}$ and $P_{y}$ envelope wave functions that comes from 

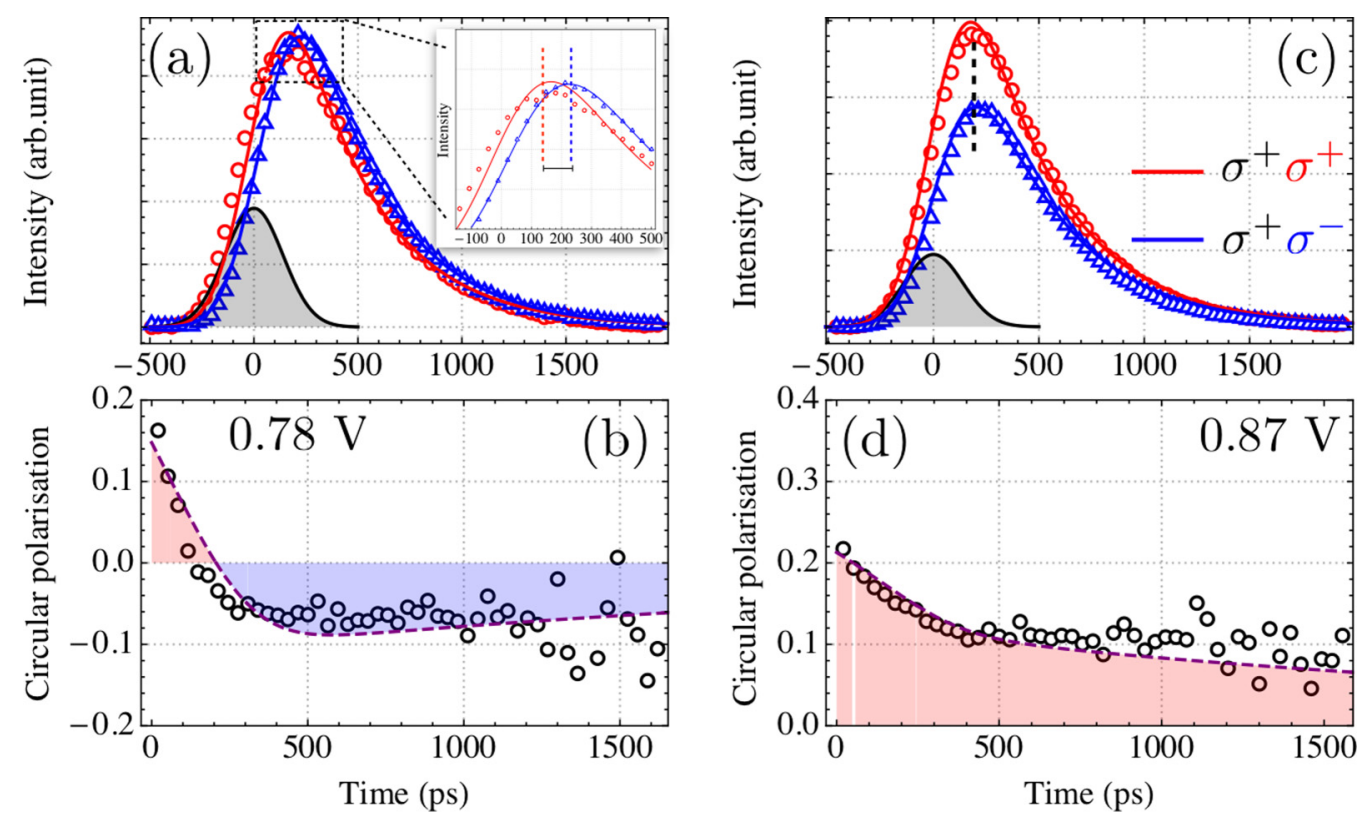

FIG. 3. (a) Time dynamics of the co- and contrapolarized luminescence of $X^{-}$when the applied bias is $0.78 \mathrm{~V}$. The laser energy is set $26 \mathrm{meV}$ above the $X^{-}$emission energy, corresponding to excitation of the $P$-shell resonance. The response of the APD to a 2 -ps pulse is added in black. The red and blue lines are fits taking into account the detector convolution. (b) Dynamics of the circular polarization $P_{c}$ of the $X^{-}$emission without deconvolution. (c) and (d) As in (a) and (b) but with applied bias of $0.87 \mathrm{~V}$.

the QD elongation perpendicular to the growth axis [12]. The fine structure of the hot trion, where one electron occupies an $S$ orbital and a second electron occupies the $P$ orbital, consists of the triplet states $T_{0}\left(\uparrow_{s} \downarrow^{p}+\downarrow^{s} \uparrow_{p}\right) / \sqrt{2}, T_{+1}\left(\uparrow_{s} \uparrow_{p}\right)$, and $T_{-1}$ $\left(\downarrow^{s} \downarrow^{p}\right)$ and a singlet $S_{0}^{*}$ state $\left(\uparrow_{s} \downarrow^{p}-\downarrow^{s} \uparrow_{p}\right) / \sqrt{2}$. The singlet state is split from the triplet states by the electron-electron exchange interaction $\Delta_{e e}$.

Contrary to the relaxed trion where all the carriers are in the $S$ shell and the electron-hole exchange interaction plays no role, for the hot trion the isotropic component of the electronhole exchange interaction lifts the degeneracy of the triplet by an amount $\tilde{\Delta}_{0}=\frac{1}{2}\left(\Delta_{0}^{S_{e}-S_{h}}+\Delta_{0}^{P_{e}-S_{h}}\right)\left[\tilde{\tilde{\Delta}}_{0}=\frac{1}{2}\left(\Delta_{0}^{S_{e}-P_{h}}+\right.\right.$ $\left.\left.\Delta_{0}^{P_{e}-P_{h}}\right)\right]$ if the hole occupies the $S$-like orbital (P-like).

The quantity $\tilde{\Delta}_{0}$ is expressed using the effective electron-hole exchange interaction Hamiltonian for $C_{2 v}$ symmetry $[13,14]$ :

$$
\hat{H}_{\mathrm{eeh}}=\sum_{i=\left(S_{e}, P_{e}\right)}-2 \Delta_{0}^{i-S_{h}} \hat{S}_{z}^{e_{i}} \hat{J}_{z}^{h}+\frac{\delta_{1}^{i-S_{h}}}{2}\left(\hat{S}_{+}^{e_{i}} \hat{J}_{-}^{h}+\hat{S}_{-}^{e_{i}} \hat{J}_{+}^{h}\right),
$$

where $\hat{S}^{e_{i}}$ is the electron spin operator occupying the $i$ orbital and $\hat{J}^{h}$ is the pseudo-1/2 spin operator for the heavy hole with the convention $\hat{J}_{z}^{h}|\Uparrow\rangle=\frac{1}{2}|\Uparrow\rangle$ and $\hat{J}_{z}^{h}|\Downarrow\rangle=-\frac{1}{2}|\Downarrow\rangle$. We neglect in Eq. (1) the anisotropic exchange splitting of the dark exciton $\delta_{2}$ as it is more than one order of magnitude weaker than the exchange splitting of the bright exciton $\delta_{1}$.

When the laser is $\sigma^{+}$polarized and tuned to the $P_{h}-P_{e}$ transition energy, i.e., $27 \mathrm{meV}$ above the trion emission energy, the bright triplet states $\left|T_{-1}, \Uparrow P_{h}\right\rangle$ and $\left|T_{0}, \Uparrow P_{h}\right\rangle$ are populated. They decay rapidly in a typical hole relaxation time $\tau_{P_{h} \rightarrow S_{h}}$ to $\left|T_{-1}, \Uparrow s_{h}\right\rangle$ and $\left|T_{0}, \Uparrow S_{h}\right\rangle$. A Pauli spin blockade prevents the $P$ electron of the $\left|T_{-1}, \Uparrow S_{h}\right\rangle$ state from relaxing to the $S$ shell without an electron-hole spin flip flop.
This electron-hole spin flip flop is due to the anisotropic part of the electron-hole exchange interaction [see Eq. (1)]. As a consequence, $\left|T_{-1}, \Uparrow P_{h}\right\rangle$ generated by $\sigma^{+}$polarized excitation relaxes via the intermediate $\mid T_{-1}$, $\left.S_{h}\right\rangle$ state to the $\left|S_{0}, \Downarrow^{S_{h}}\right\rangle$ trion state. As shown by the blue path drawn in Fig. 2, this relaxation pathway ultimately leads to emission from the negative trion $X^{-}$which is contrapolarized with respect to the laser excitation.

The efficiency of the electron-hole spin flip-flop mechanism which leads to contrapolarized emission can be measured by time-resolved photoluminescence experiments [15]. Figure 3(a) shows the dynamics of the co- and contrapolarized components of the $X^{-}$photoluminescence following a 2-ps circularly polarized laser pulse. The laser energy is $1.761 \mathrm{eV}$, i.e., within the $P_{h}-P_{e}$ transition, and the applied bias is $0.78 \mathrm{~V}$. The PL signal is recorded by a $\mathrm{Si}$ avalanche photodiode (APD) with a Gaussian time response at FWHM of 240 ps (see Appendix A). After 200 ps, the contrapolarized emission intensity is greater than the copolarized one, indicating that the degree of circular polarization $P_{c}$ becomes negative. The inset in Fig. 3(a) shows a zoom for a time window centered at 200 ps. We observe that the maximum intensity of the copolarized intensity is reached at $\sim 160 \mathrm{ps}$, whereas for contrapolarized intensity, the maximum is located at $\sim 220 \mathrm{ps}$. The contrapolarized intensity delay time is clearly associated with the flip-flop process effective time $\tau_{f f}$ (see Fig. 2). Using a simple rate equation model and taking into account the APD time response, we estimate that $\tau_{f f}=70 \mathrm{ps}$ (see Appendix B). This value is about four times faster than the contrapolarized PL component delay time observed in InAs [2] and InP QDs ensembles [15].

In more detail, three mechanisms, reliant on the electronhole anisotropic exchange interaction, have been invoked to explain the relaxation of the Pauli-blocked $\left|T_{-1}, \Uparrow S_{h}\right\rangle$ state 
to the $\left|S_{0}, \Downarrow^{S_{h}}\right\rangle$ trion state [6]. The first mechanism relies on a combination of electron-hole and electron-phonon interactions to couple $\left|T_{-1}, \Uparrow S_{h}\right\rangle$ to the virtual state $\left|S_{0}, \Downarrow^{S_{h}}+1 \mathrm{LO}\right\rangle$. By treating this latter interaction in the framework of the Fröhlich model, it was shown in Ref. [7] that this mechanism, which successfully described the negative circular polarization dynamics of the trion of a single InAs QD, becomes ineffective for a large mismatch between the $S$ - $P$ splitting energy and the LO phonon energy [16]. This is also the case for the GaAs/AlAs dots studied here, where the $S$ - $P$ splitting energy is $\sim 16 \mathrm{meV}$ (see Fig. 2), compared to the $36-\mathrm{meV}$ LO phonon energy in GaAs. The second mechanism is based on coupling between the $\left|T_{-1}, \Uparrow S_{h}\right\rangle$ and $\left|S_{0}^{\star}, \Downarrow^{S_{h}}\right\rangle$ states, followed by thermalization to the $X^{-}\left(\left|S_{0}, \Downarrow^{S_{h}}\right\rangle\right)$ state [2]. In this case, the spin flip-flop rate $\tau_{f f}^{-1}$ is proportional to $\frac{\left(\delta_{1}^{S_{h}-S_{e}}-\delta_{1}^{S_{h}-P_{e}}\right)^{2}}{\left(\Delta_{e e}-\tilde{\Delta}_{0}\right)^{2}}$. Since the electron exchange interaction energy $\Delta_{e e}$ is in the meV range, $\tau_{f f}$ has been estimated to be of the order of nanoseconds [7,17]. This is much longer than the value of $\tau_{f f}$ experimentally observed here. In contrast, the third mechanism is based on coupling between the $\mid T_{-1}$, $\left.s_{h}\right\rangle$ and $\left|T_{0}, \Downarrow^{S_{h}}\right\rangle$ states followed by thermalization to the $\left|S_{0}, \Downarrow^{S_{h}}\right\rangle$ state [6]. In this case, $\tau_{f f}^{-1}$ is proportional to $\frac{\left(\delta_{1}^{S_{h}-S_{e}}+\delta_{1}^{S_{h}-P_{e}}\right)^{2}}{\tilde{\Delta}_{0}^{2}}$. Since $\tilde{\Delta}_{0}$ is of the order of $0.1 \mathrm{meV}$, this will give a much shorter flip-flop lifetime than the previous mechanism. The subsequent thermalization of $\left|T_{0}, \Downarrow^{S_{h}}\right\rangle$ to the $X^{-}$state was found to be $\sim 10 \mathrm{ps}$ [6], only one order of magnitude greater than for $\left|S_{0}^{\star}, \Downarrow^{S_{h}}\right\rangle$, due to a spin-orbit-induced asymmetric electron-electron interaction between $\left|T_{0}, \Downarrow^{S_{h}}\right\rangle$ and $\left|S_{0}^{\star}, \Downarrow^{S_{h}}\right\rangle$ $[6,18,19]$. This relaxation mechanism is therefore compatible with the experimental determination of $\tau_{f f}$ of $70 \mathrm{ps}$ here.

Figure 3(b) shows the dynamics of the degree of circular polarization of $X^{-}$emission. The experimental points are plotted using the data shown in Fig. 3(a), i.e., without any deconvolution procedure. We observe a long decay time of the negative circular polarization. From this decay time we evaluate the effective hole spin lifetime $\tau_{s}^{h}$ to be $2.7 \mathrm{~ns}$ (see Appendix B). This is the first time that the hole spin lifetime has been measured in such GaAs/AlAs QDs and indicates that it is relatively short. For comparison, similar measurements of the InAs/GaAs QD ensemble gave $\tau_{s}^{h} \gtrsim 20 \mathrm{~ns}$ [20].

Figures 3(c) and 3(d) show the effect of a small change in applied bias on the $X^{-}$polarization while keeping the same laser excitation energy. With an applied bias of $0.87 \mathrm{~V}$, there is no delay between the contra- and copolarized components, with both having their maximum intensity at $\sim 180 \mathrm{ps}$, as shown by the vertical dashed line in Fig. 3(c). Figure 3(d) shows that this lack of delay means that there is no negative degree of circular polarization at this bias voltage.

At this stage, two conclusions arise from these observations. First, since increasing (decreasing) the bias is able to decrease (increase) the relative fraction of contrapolarized emission, it must be inducing a weakening (enhancing) of the relative absorption of the $\left|T_{-1}, \Uparrow P_{h}\right\rangle$ state compared to the $\left|T_{0}, \Uparrow P_{h}\right\rangle$ state. Second, there is no measurable delay between the contrapolarized and copolarized emissions for a bias voltage of $0.87 \mathrm{~V}$ (compared to the 70-ps delay of the contrapolarized emission at $0.78 \mathrm{~V}$ ). The fraction of emission that is contrapolarized, however, remains significant
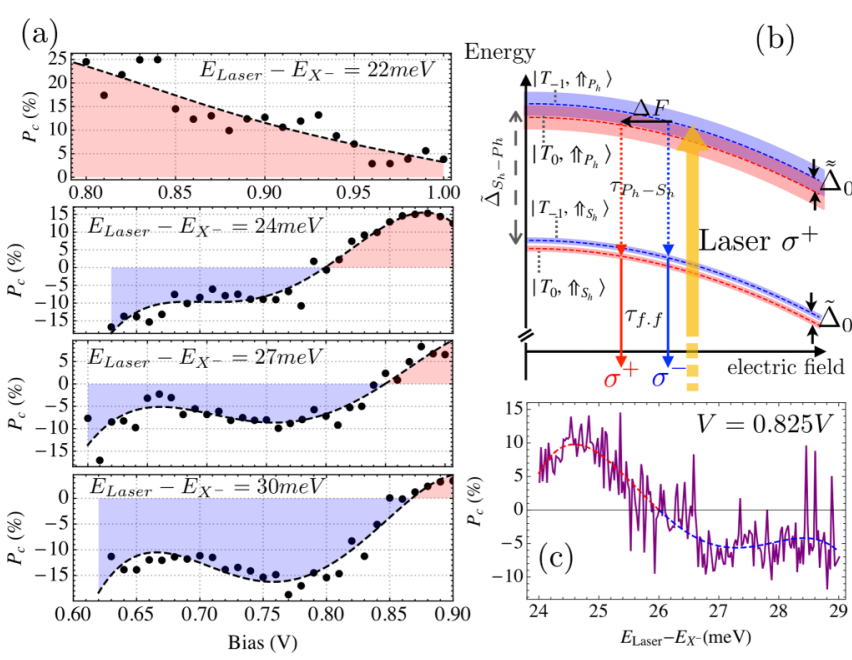

FIG. 4. (a) Voltage scans of the $X^{-}$emission circular polarization for different excitation energies within the $P$-shell resonance. The black dashed lines are guides to the eye. (b) Schematic showing the change in polarized emission from contrapolarized (blue) to copolarized (red) on changing the electric field across the QD by $\Delta F$ (change in applied bias $\Delta V>0$ ). The electric-field-induced energy shift of the QD states relative to the exciting laser energy leads to a decrease in absorption of the $\left|T_{-1}, \Uparrow P_{h}\right\rangle$ state compared to $\left|T_{0}, \Uparrow P_{h}\right\rangle$ (see the text for a detailed explanation). (c) Circular-polarizationresolved PLE of $X^{-}$with a laser step of $11 \mu \mathrm{eV}$. The dashed red and blue lines are guides to the eye. The apparent noise in $P_{c}$ comes from fluctuations in the electric field at this applied voltage, observable as fluctuations in the photocurrent during the PLE measurement.

[Figs. 3(c) and 3(d)], with an $\sim+20 \%$ positive degree of circular polarization. The lack of delay time indicates that this contrapolarized emission is not due to the spin flip-flop relaxation of the $\left|T_{-1}, \Uparrow S_{h}\right\rangle$ state to the $\left|S_{0}, \Downarrow^{S_{h}}\right\rangle$ state. A fast hole spin relaxation time in the $P$ orbital would, however, lead to rapid filling of both the $\left|T_{0}, \Uparrow P_{h}\right\rangle$ and $\left|T_{0}, \Downarrow^{P_{h}}\right\rangle$ states after excitation with positive circularly polarized light [21], providing a possible route to lower the degree of circular polarization. This hole spin relaxation in the $P$ shell prior to thermal relaxation to the $S$ shell would also contribute to decreasing the amplitude of the negative degree of circular polarization at $0.78 \mathrm{~V}$ [see Fig. 3(a)]. Under this voltage condition, where the $\left|T_{-1}, \Uparrow P_{h}\right\rangle$ state is being filled preferentially to the $\left|T_{0}, \Uparrow P_{h}\right\rangle$ state, hole spin relaxation in the $P$ orbital would reduce the fraction of the $\left|T_{-1}, \Uparrow P_{h}\right\rangle$ state due to filling the $\left|T_{-1}, \Downarrow^{P_{h}}\right\rangle$ state. This would subsequently reduce the contrapolarized emission that is due to the spin flip flop of the $\left|T_{-1}, \Uparrow s_{h}\right\rangle$ state followed by thermal relaxation of the $\left|T_{0}, \Downarrow^{S_{h}}\right\rangle$ state.

This assumption of fast hole spin relaxation in the $P$ orbital is used in the calculations presented in Appendix B, where $\tau_{f f}$ is quantified as $70 \mathrm{ps}$, taking into account the APD temporal response.

To study further the effect of applied bias on the relative absorption of the different triplet states, Fig. 4(a) shows the $X^{-}$degree of circular polarization using a cw spectrally narrow laser tuned to slightly different energies within the $P$-shell electronic resonance. For a fixed excitation energy, the degree of circular polarization $P_{c}$ changes from negative to positive 
(contrapolarized to copolarized) with increasing bias voltage. However, with increasing laser energy, the voltage range for which $P_{c}$ is negative increases, while both the voltage range and the magnitude of positive $P_{c}$ decreases. The critical excitation energies for which $P_{c}$ will remain positive (negative) over the entire voltage range of the $X^{-}$emission are found experimentally to be $E_{\text {Laser }}-E_{X^{-}} \lesssim 22 \mathrm{meV}$ ( $\left.\gtrsim 30 \mathrm{meV}\right)$.

The combined effect of excitation energy and applied bias on the presence or absence of a negative degree of circular polarization can be explained by considering the Stark shift of the QD energy levels with applied bias and the induced effect on the probability of absorption of different triplet states. This is shown schematically in Fig. 4(b). When the $\sigma^{+}$polarized laser energy is high enough, the absorption on the higherenergy $\left|T_{-1}, \Uparrow P_{h}\right\rangle$ state will be greater than that of the $\left|T_{0}, \Uparrow P_{h}\right\rangle$ state (see Fig. 2). Fast relaxation from the $\left|T_{-1}, \Uparrow P_{h}\right\rangle$ state to the $\left|T_{-1}, \Uparrow_{S_{h}}\right\rangle$ state leads to contrapolarized $X^{-}$emission, as displayed by the blue arrows in Fig. 4(b). Increasing the bias leads to a decrease of the electric field across the dots and a blue Stark shift of the optical transitions [22]. When the Stark shift is sufficiently large to bring the $\left|T_{0}, \Uparrow P_{h}\right\rangle$ state closer into resonance with the excitation laser energy than the $\left|T_{-1}, \Uparrow P_{h}\right\rangle$ state, absorption of the $\left|T_{0}, \Uparrow P_{h}\right\rangle$ state will dominate. Relaxation from this state leads to copolarized emission of $X^{-}$, as shown by the red lines in Fig. 4(b).

Because of the large broadening of the $P$-shell resonance [Fig. 1 (b)], the transition from absorption in the $\left|T_{-1}, \Uparrow_{P_{h}}\right\rangle$ state to the $\left|T_{0}, \Uparrow S_{h}\right\rangle$ state when changing the laser energy is not abrupt.

To illustrate this, Fig. 4(c) shows the circular-polarizationresolved PLE over a small spectral window around the transition from positive to negative $P_{c}$ at fixed bias $(0.825 \mathrm{~V})$. When the laser is tuned to the lower-energy side, $P_{c}$ is positive, whereas on the higher-energy side, $P_{c}$ is negative, which is consistent with the higher energy of $T_{-1}$ compared to $T_{0}$. The sign of $P_{c}$ crosses from positive to negative at $E_{\text {Laser }}-E_{X^{-}} \sim$ $26 \mathrm{meV}$. This corresponds to the point where absorption and recombination of the $T_{-1}$ and $T_{0}$ states are balanced, giving similar co- and contratrion intensities. If the laser energy is set too far above or below this position, the amplitude of the Stark shift over the voltage range of the stability of $X^{-}$ is not enough to change the absorption from $T_{-1}$ to $T_{0}$ (or vice versa), and there will subsequently be no change in sign of $P_{c}$ on changing the bias. This situation is illustrated in Fig. 4(a) for $E_{\text {Laser }}-E_{X^{-}}=22 \mathrm{meV}$ and, to some extent, for $E_{\text {Laser }}-E_{X^{-}}=30 \mathrm{meV}$.

When looking carefully at Fig. 4(a), we observe that the maximum positive $P_{c}$ reaches a value greater than $+25 \%$, whereas the maximum negative $P_{c}$ seems to be pinned at $\sim-$ $15 \%$. Under excitation and bias conditions where absorption can be assumed to be predominantly either in the $\left|T_{-1}, \Uparrow P_{h}\right\rangle$ or $\left|T_{0}, \Uparrow_{P_{h}}\right\rangle$ state, $P_{c}$ of $X^{-}$will reflect spin relaxation occurring as the hot trion states relax down to either the $\left|S_{0}, \Downarrow^{S_{h}}\right\rangle$ or $\left|S_{0}, \Uparrow s_{h}\right\rangle$ state (Fig. 2). If $P_{c}$ were being limited by spin relaxation of the hole in the $P$ orbital prior to its relaxation to the $S$ shell, then this would lead to a reduction of the maximum $P_{c}$ for both co- and contrapolarized components of the PL in a similar way.

To understand the lower amplitude for the negative $P_{c}$, we set the laser energy to the position of the $S_{h}-P_{e}$ transition while
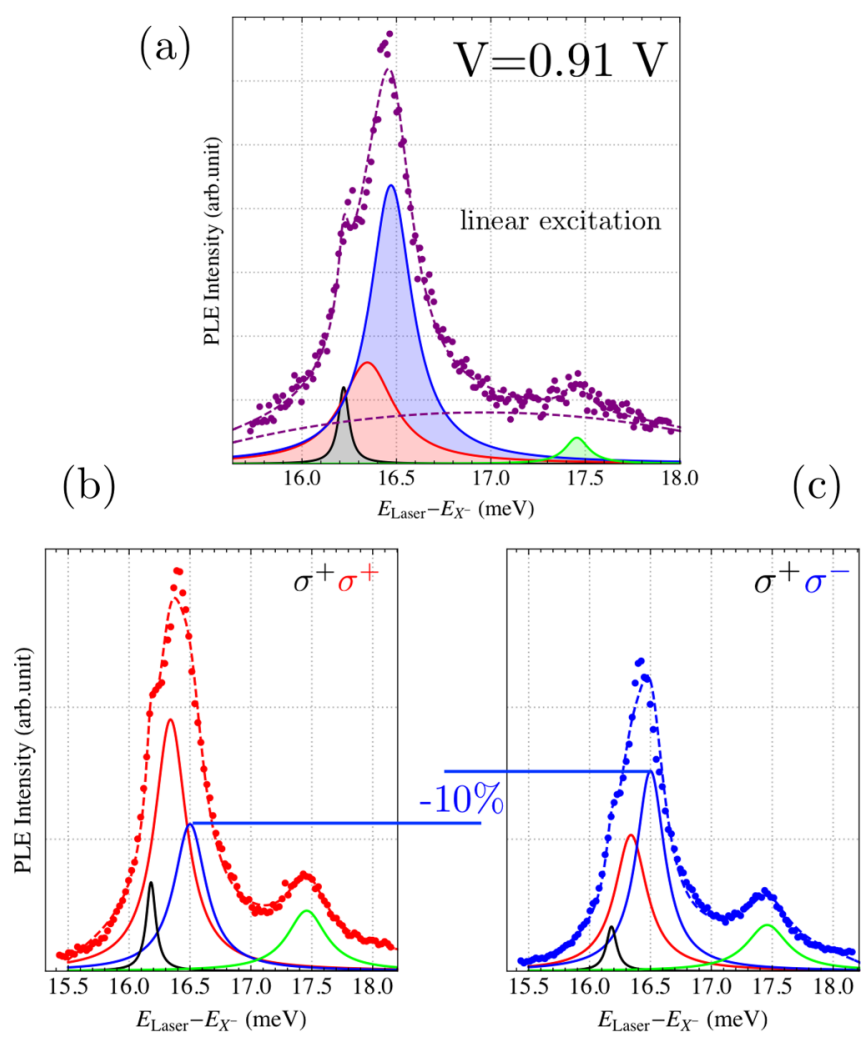

FIG. 5. (a) $X^{-}$PLE spectra when the laser energy is scanning the $S_{h}-P_{e}$ transition. The laser is linearly polarized and no-polarization selective detection is employed. The PLE lines are fitted by including a local quadratic background shown by the purple dashed curve. (b) and (c) The same experiment setting the laser polarization $\sigma^{+}$and analyzing (b) $\sigma^{+}$and (c) $\sigma^{-}$. The laser intensity in (b) and (c) is twice that used in (a) to enhance the signal-to-noise ratio when measuring the circular polarization of the hot trion triplet components.

recording the PL signal from the $X^{-}$line. In principle, the $S_{h}-P_{e}$ optical transition is not allowed due to the parity of the envelope wave functions; however, the low symmetry of these QDs fabricated by nanohole infilling efficiently relaxes the optical selection rules [23].

Figure 5(a) shows the PLE for $X^{-}$while the linearly polarized laser scans the $S_{h}-P_{e}$ transition $\sim 16 \mathrm{meV}$ above the trion energy. The laser intensity was reduced as much as possible while still maintaining an acceptable signal-to-noise ratio. The amplitude of the PLE signal is seven times weaker than the signal obtained when exciting the $P$-shell resonance [Fig. 1(b)]. We observe a complex multipeak structure appearing for $E_{\text {Laser }}-E_{X^{-}} \sim 16.5 \mathrm{meV}$. This can be fitted satisfactorily by three equidistant Lorentzian lines which correspond to the absorptions of $\left|T_{-1}, \Uparrow_{S_{h}}\right\rangle$ and $\left|T_{+1}, \Downarrow^{S_{h}}\right\rangle$ at high energy (in blue in Fig. 5), $\left|T_{0}, \Uparrow S_{h}\right\rangle$ and $\left|T_{0}, \Downarrow^{S_{h}}\right\rangle$ (in red), and the "dark" hot trion configurations $\left|T_{+1}, \Uparrow S_{h}\right\rangle$ and $\left|T_{-1}, \Downarrow^{S_{h}}\right\rangle$ (in black) at low energy. The additional, well-separated, weak electronic resonance observable at $17.5 \mathrm{meV}$ is assigned to absorption of the singlet states $\left|S_{0}^{\star}, \Uparrow_{h}\right\rangle$ and $\left|S_{0}^{\star}, \Downarrow^{S_{h}}\right\rangle$.

This result differs from similar measurements performed on InAs/GaAs QDs where only the bright triplet states were observed [6]. Before discussing in detail the reason for the appearance of the hot trion dark state 
configuration, we first here confirm the consistency of our interpretation.

(i) From the assignment of the peaks in Fig. 5 we are able to quantify $\tilde{\Delta}_{0}=135 \mu \mathrm{eV}$ [24] and $\Delta_{e e}=1.1 \mathrm{meV}$. The magnitude of the splitting $\Delta_{e e}$ between $T_{0}$ and $S_{0}^{\star}$ for this GaAs/AlAs QD is confirmed by PL spectroscopy measurements on the same dot where $\Delta_{e e} \sim 1 \mathrm{meV}$ is evaluated from the separation of the singlet-triplet components of the $X^{2-}$ state (see Appendix A). It should be noted that this value is much weaker than in InAs/GaAs QDs, where it lies in the range of $\sim 5 \mathrm{meV}[6,25]$.

(ii) The ratio of the oscillator strength between the two bright triplet states $r=\frac{f_{\left|T_{-1}, \Uparrow_{h}\right\rangle}}{f_{\left|T_{0}, s_{S_{h}}\right\rangle}}$ is expected to be equal to 2 $[6,16,26,27]$. We find that the ratio of the integrated area of blue and red Lorentzian curves is 2.2, in good agreement with the expected value. As pointed out in Ref. [6], these absorption peaks are broadened when the laser power is enough to saturate the transitions. By repeating the same experiments shown in Fig. 5(a) using an excitation power two times more intense (not shown), we measure the same width of the PLE lines, indicating that they are already close to the saturation regime in Fig. 5(a). As a consequence, the linewidths of the Lorentzian fits are much greater than the natural linewidth due to laser-power-induced broadening, which explains the overlap of the different PLE lines.

(iii) Circular-polarization-resolved PLE obtained when the laser is $\sigma^{+}$polarized [Figs. 5(a) and 5(b)] confirmed that only the PLE line associated with the $\left|T_{-1}, \Uparrow s_{h}\right\rangle$ state is contrapolarized with $P_{c}=-10 \%$. To calculate the degree of circular polarization we use here the integrated areas from the $\sigma^{+} \sigma^{+}$and $\sigma^{+} \sigma^{-}$PLE spectra, i.e., $I_{\sigma^{+} \sigma^{+}}$and $I_{\sigma^{+} \sigma^{-}}$. The same analysis gives a circular polarization of $+30 \%$ for both $T_{0}$ and the dark PLE lines and a value of $+10 \%$ for $S_{0}^{\star}$. The circular polarization of $S_{0}^{\star}$ is surprisingly weak given the long hole spin relaxation time $(2.7 \mathrm{~ns})$, although the positive sign is the expected one. It is possible that the large background in the PLE signal due to the high power needed for the measurement is leading to an underestimation of this value. Note that similar experiments performed on InAs QDs have shown that the degree of circular polarization obtained for the $\left|T_{-1}, \Uparrow S_{h}\right\rangle$ PLE line exceeds $-95 \%$, in stark contrast to our result. We show next that the modest degree of negative circular polarization and the presence of the dark hot trion PLE line are strongly related.

The optical activity of the dark spin configuration exciton results from the low $C_{s}$ symmetry of the QD confinement potential $[9,28,29]$. This symmetry breaking is taken into account in the effective Hamiltonian by adding new contributions to $\hat{H}_{\text {eeh }}$ in Eq. (1) $[9,28]$ as

$$
\delta \hat{H}_{\mathrm{eeh}}^{C_{s}}=\sum_{i=\left(S_{e}, P_{e}\right)} \delta_{11}^{i-S_{h}} \hat{J}_{z}^{h}\left(\hat{S}_{+}^{e_{i}}+\hat{S}_{-}^{e_{i}}\right)+\delta_{12}^{i-S_{h}} \hat{S}_{z}^{e_{i}}\left(\hat{J}_{+}^{h}+\hat{J}_{-}^{h}\right)
$$

where $\delta_{11}^{i-S_{h}}$ and $\delta_{12}^{i-S_{h}}$ are the energies related to the noncollinear terms coupling bright-to-dark excitonic configurations by flipping solely the spin of the electron occupying the orbital $i$ or hole on the $S_{h}$ orbital. Treating similarly $\delta \hat{H}_{\mathrm{eeh}}^{C_{s}}$ and the anisotropic part of $\hat{H}_{\text {eeh }}$, perturbation theory gives, to first order, the dark hot trion state as

$$
\left|\widetilde{T_{+1}, \Uparrow S_{h}}\right\rangle=\left|T_{+1}, \Uparrow s_{h}\right\rangle-\alpha\left|T_{0}, \Uparrow s_{h}\right\rangle,
$$

and for the higher-energy triplet state it gives

$$
\left|\widetilde{T_{-1}, \Uparrow s_{h}}\right\rangle=\left|T_{-1}, \Uparrow s_{h}\right\rangle+\beta\left|T_{0}, \Downarrow^{S_{h}}\right\rangle+\alpha\left|T_{0}, \Uparrow s_{h}\right\rangle,
$$

with $\alpha=\frac{1}{2 \sqrt{2}}\left[\frac{\delta_{11}^{S_{e}-S_{h}}+\delta_{11}^{P_{e}-S_{h}}}{\tilde{\Delta}_{0}}\right]$ and $\beta=\frac{1}{2 \sqrt{2}}\left[\frac{\delta_{1}^{S_{e}-S_{h}}+\delta_{1}^{P_{e}-S_{h}}}{\tilde{\Delta}_{0}}\right]$. In Eqs. (3) and (4), we have kept the dominant terms in $\tilde{\Delta}_{0}^{-1}$ and have neglected terms with energy denominators $2 \tilde{\Delta}_{0}$ [30] and $\Delta_{e e}$ from the perturbative expansion.

Equation (3) shows that the dark triplet state is mixed with the bright $\mid T_{0}$, $\left.S_{h}\right\rangle$, with a coupling $\alpha$ transferring oscillator strength to the dark configuration. Equation (3) also predicts that the dark state should have the same circular polarization sign as the $\left|T_{0}, \Uparrow s_{h}\right\rangle$ state, in agreement with the experimental observations from the PLE measurements. As a consequence, $\alpha^{2}$ quantifies the ratio of the oscillator strengths of the bright and dark states, which can be evaluated to first order as the ratio of the integrated areas of the PLE lines in Fig. 5(a) and is equal to 0.13 . Assuming $\delta_{11}^{S e-S h} \sim \delta_{11}^{P e-S h}$, we obtained $\delta_{11}^{S e-S h} \sim 70 \mu \mathrm{eV}$, in very good agreement with magnetospectroscopy experiments performed on similar QDs [31].

Equation (4) describes the effect of the QD asymmetry on the $\left|T_{-1}, \Uparrow S_{h}\right\rangle$ state. The first term gives the coupling between $\left|T_{-1}, \Uparrow_{S_{h}}\right\rangle$ and $\left|T_{0}, \Downarrow^{S_{h}}\right\rangle$. This coupling is governed by the anisotropic exchange splittings present in $\beta$ and is the origin of the negative circular polarization. The second term describes an additional coupling to $\left|T_{0}, \Uparrow s_{h}\right\rangle$, weighted by $\alpha$. This term comes from including $\delta \hat{H}_{\text {eeh }}^{C_{s}}$, and it acts to offset the coupling to the $\left|T_{0}, \Downarrow^{S_{h}}\right\rangle$ state, thereby reducing the value of the negative $P_{c}$. In other words, when the condition $\beta^{2} \gg \alpha^{2}$ is fulfilled, the negative circular polarization could be close to $-100 \%$, as already reported for InAs/GaAs QDs [6], but as $\alpha$ becomes more significant, the negative circular polarization is reduced.

The maximum achievable negative polarization measured in cw experiments can be expressed by $P_{c}^{\lim }=\frac{\alpha^{2}-\beta^{2}}{\alpha^{2}+\beta^{2}}$. In order to evaluate $P_{c}^{\mathrm{lim}}, \alpha$ can be experimentally determined from the relative PLE intensity of the dark and bright trion states, as previously discussed. Estimation of $\beta^{2}$ can be carried out by using the simplification $\delta_{1}^{P_{e}-S_{h}} \sim \delta_{1}^{S_{e}-S_{h}}$, with $\delta_{1}^{S_{e}-S_{h}}$ being the fine-structure splitting of the neutral exciton measured to be $95 \mu \mathrm{eV}$ (see Appendix A). We find then $\beta^{2}=0.24$ and thus $P_{c}^{\mathrm{lim}}=-30 \%$, which is confirmed experimentally. The validity of the expression defining $P_{c}^{\mathrm{lim}}$ relies on the fact that the splittings between $T_{-1}$ and $T_{0}$ with hole spin both up and down are the same, and the thermalizations of both $T_{0}$ towards the ground trion states are identical. Taking into account all the perturbative terms in Eq. (4) would lead to nontrivial expression of $P_{c}^{\text {lim }}$ and may explain the difference between the theoretical value of $P_{c}^{\mathrm{lim}}$ and the maximum experimental negative degree of polarization of $\sim-15 \%$. It should be noted that the limitation of the degree of negative circular polarization is linked to the nanohole etching conditions used, which yielded relatively deep and wide nanoholes that had an asymmetric profile along [ -110$]$ and that were only partially infilled. Other work has shown that it is possible to have high-symmetry GaAs/AlAs QDs in nanoholes with the use 
of smaller initial droplets, leading to less deep nanoholes, annealing under an $\mathrm{As}_{2}$ overpressure, and complete hole infilling with GaAs [32]. This means that further optimization of the diode structure and dot growth conditions should improve the degree of negative circular polarization observed here.

This simple model, based on the effect of symmetry breaking of the QD confinement potential, explains simultaneously the appearance of absorption of the dark spin configuration hot trion and the reduction of negative circular polarization of $X^{-}$.

\section{CONCLUSION}

In this paper, we demonstrated electrical control of the exciton charge state of a GaAs/AlAs quantum dot fabricated by in situ droplet etching of nanoholes and partial infilling. Using $P$-shell excitation, electrical control of the degree of circular polarization of the $X^{-}$state, going continuously from positive (copolarized) to negative (contrapolarized), was demonstrated. This control over the sign of the degree of circular polarization is due to a modification of the relative occupation of the hot triplet states either by the Stark shift or by the laser excitation energy within the $P$-shell resonance. This voltage dependence means that modulation of the bias component, while remaining within the stability region of the negative trion, could be used to provide on-demand control of the sign of the circular polarization from a single quantum emitter. This has potential application in quantum information protocols.

A higher degree of negative polarization can be achieved by optimization of the diode structure to increase the stability region of the negative trion together with improvement in the growth conditions during nanohole formation and infilling to give QDs with higher symmetry.

\section{ACKNOWLEDGMENTS}

This work was financially supported by the French National Research Agency (ANR "SPINEX" ANR-17-CE300022-01 and ANR "ISQUAD" ANR-18-CE47-0006-01), by the Paris Île-de-France Région in the framework of DIM SIRTEQ, and by the Cluster of Excellence MATISSE led by Sorbonne Universités. We thank C. Manquest for technical support.

\section{APPENDIX A: EXPERIMENTAL ESTIMATION OF $\Delta_{e}$ FOR A SINGLE GaAs/AIAs QD FABRICATED BY NANOHOLE INFILLING}

We present here the experiments which provide quantitative measurements of the energy splittings used in the main text. All the experiments were performed on the same QD used previously with green laser excitation at a temperature of $4 \mathrm{~K}$.

When the green laser excites the QD, new lines associated with a highly charged exciton appear when the applied bias is inside the domain of existence of $X^{-}$(see Fig. 1). In order to confirm the estimation of $\Delta_{e e}$ done using PLE experiment via the $S_{h}-P_{e}$ transition, the optical spectroscopy of the doubly charged exciton stands as a good tool for estimating $\Delta_{e e}$,

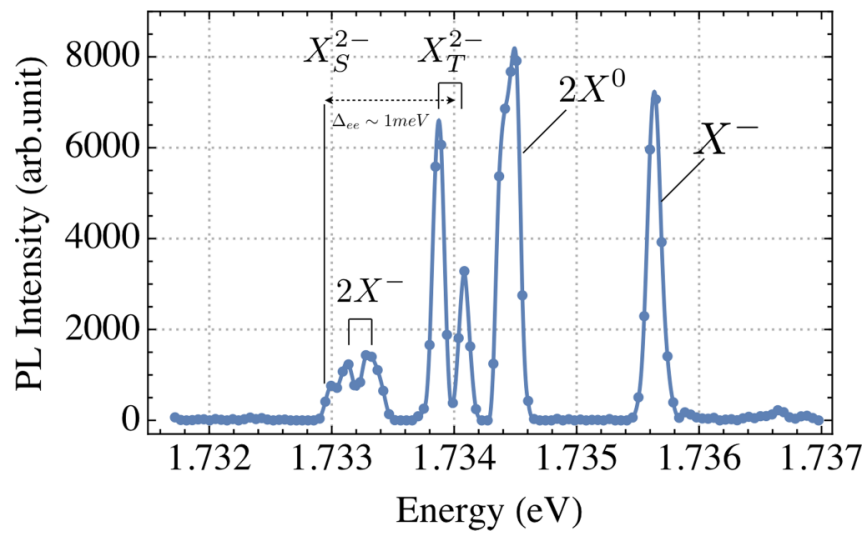

FIG. 6. Photoluminescence spectrum from the studied QD showing the fine structure of $X^{2-}$.

provided both triplet and singlet components can be detected [25]. In Fig. 6, we distinguish the weak PL associated with the singlet appearing on the low-energy side of the charged biexciton $2 X^{-}$, as well as the two triplet components. This provides an estimation of $\Delta_{e e}$ of $1 \mathrm{meV}$, in good agreement with the experimental determination given in Fig. 5(a).

Also, in Fig. 7 we show the experimental result leading to the estimation of $\delta_{1}^{S_{e}-S_{h}}$. We analyze the neutral exciton $X^{0}$ emission energy position as a function the linear polarization by rotating a $\lambda / 2$-wave plate placed before the linear polarizer and from the amplitude of the sinusoidal fit we extract $\delta_{1}^{S_{e}-S_{h}}$ of $\sim 95 \mu \mathrm{eV}$.

\section{APPENDIX B: ANALYSIS OF THE CIRCULAR POLARIZATION DYNAMICS}

The objective of this Appendix is to present a consistent determination of $\tau_{f f}$ giving the experimental result in Fig. 3. This requires, first, calculating the PL dynamics using a simple rate equation model [15] and, second, taking into account the time response of the APD.

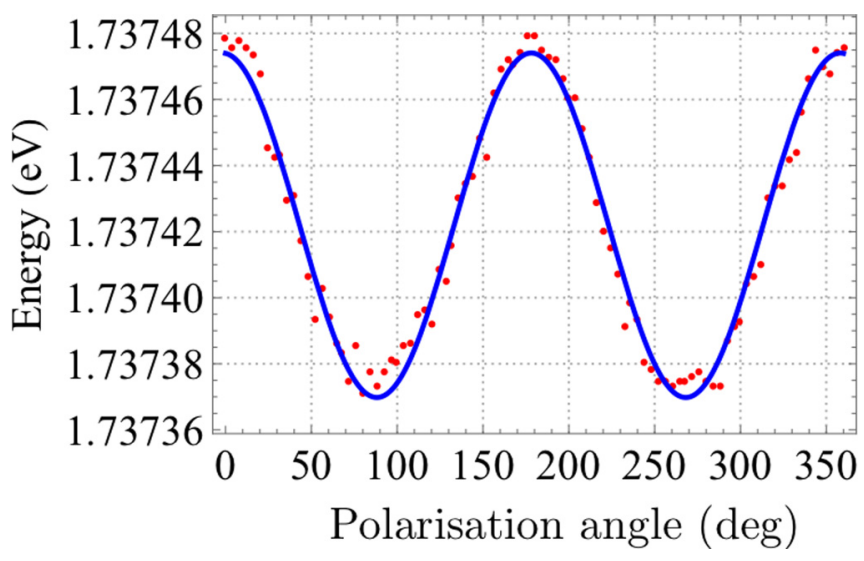

FIG. 7. Neutral exciton emission energy as a function of polarization angles (red points). A fine-structure splitting of $\sim 95 \mu \mathrm{eV}$ is deduced from the sinusoidal fit (blue line). 

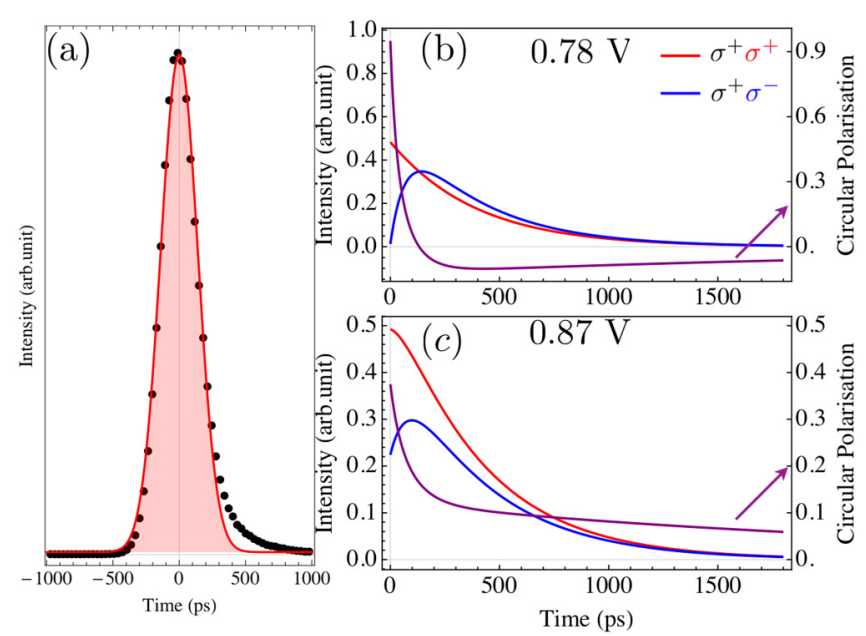

FIG. 8. (a) Gaussian response of the APD to a 2-ps laser pulse excitation. The red curve is a fit. We discard in the calculations the weak discrepancy at long-delay positive time where the intensity is very weak. (b) and (c) Deconvoluted signals corresponding to the experimental data presented in Fig. 3.

\section{APD temporal response}

The APD response is shown in Fig. 8(a) when a 2-ps laser pulse is sent to the detector. We extract then a Gaussian response $G(t)$ with a FWHM of $240 \mathrm{ps}$.

The intensity signal $C(t)$ measured by the detector is then equal to the convolution $G(t) \star[H(t) S(t)]$, where $H(t)$ is the Heaviside distribution and $S(t)$ is the deconvoluted PL signal.

\section{Rate equation model for the PL dynamics}

We present here the rate equation model used to fit the PL time dynamics in Fig. 2 when the laser is tuned to the $P$-shell resonance, considering the following reasonable assumptions: (i) the hole relaxation time from the $P$ to $S$ orbital is extremely fast, $\tau_{P_{h} \rightarrow S_{h}} \sim 1 \mathrm{ps}$, (ii) the relaxation of the triplet $T_{0}$ towards the trion is much faster than $\tau_{f f}$ for the triplet $T_{-1}$, (iii) all the bright triplet states with hole spin up or down can be populated after the hole thermalization due to the possible fast hole spin relaxation time when the hole occupies the $P$ level [33] or due to the fact that the broad picosecond-pulse $\sigma^{+}$laser can excite both $\left|T_{-1}, \Uparrow P_{h}\right\rangle$ and $\left|T_{0}, \Uparrow P_{h}\right\rangle$, and (iv) we neglect the role of the dark spin hot trion configuration.

This leads to

$$
\begin{aligned}
& \dot{n}_{\left|S_{0}, \Uparrow S_{h}\right\rangle}=-\frac{n_{\left|S_{0}, \Uparrow S_{h}\right\rangle}}{\tau_{\text {rad }}}-\frac{n_{\left|S_{0}, \Downarrow S_{h}\right\rangle}-n_{\left|S_{0}, \Uparrow s_{h}\right\rangle}}{2 \tau_{h}^{s}}+\frac{n_{\left|T_{+1}, \Downarrow S_{h}\right\rangle}}{\tau_{f f}}, \\
& \dot{n}_{\left|S_{0}, \Downarrow S_{h}\right\rangle}=-\frac{n_{\left|S_{0}, \Downarrow S_{h}\right\rangle}}{\tau_{\mathrm{rad}}}-\frac{n_{\left|S_{0}, \Uparrow s_{h}\right\rangle}-n_{\left|S_{0}, \Downarrow S_{h}\right\rangle}}{2 \tau_{h}^{s}}+\frac{n_{\left|T_{-1}, \Uparrow s_{h}\right\rangle}}{\tau_{f f}}, \\
& \dot{n}_{\left|T_{+1}, \Downarrow S_{h}\right\rangle}=-\frac{n_{\left|T_{+1}, \Downarrow S_{h}\right\rangle}}{\tau_{f f}}, \\
& \dot{n}_{\left|T_{-1}, \Uparrow S_{h}\right\rangle}=-\frac{\left.n_{\left|T_{-1}, \Uparrow s_{h}\right\rangle}\right\rangle}{\tau_{f f}},
\end{aligned}
$$

where $\tau_{\text {rad }}$ is the radiative lifetime of the trion.

The initial conditions according to assumptions (i) and (ii) are defined as

$$
\begin{gathered}
n_{\left|S_{0}, \Uparrow S_{h}\right\rangle}(t=0)=n_{\left|T_{0}, \Uparrow P_{h}\right\rangle}(t=0), \\
n_{\left|S_{0}, \Downarrow S_{h}\right\rangle}(t=0)=n_{\left|T_{0}, \Downarrow P_{h}\right\rangle}(t=0), \\
n_{\left|T_{-1}, \Uparrow S_{h}\right\rangle}(t=0)=n_{\left|T_{-1}, \Uparrow P_{h}\right\rangle}(t=0), \\
n_{\left|T_{+1}, \Downarrow S_{h\rangle}\right\rangle}(t=0)=n_{\left|T_{+1}, \Downarrow P_{h\rangle}\right\rangle}(t=0),
\end{gathered}
$$

and

$$
\begin{aligned}
& n_{\left|T_{+1}, \Downarrow P_{h}\right\rangle}(t=0)+n_{\left|T_{-1}, \Uparrow_{P_{h}}\right\rangle}(t=0) \\
& \quad+n_{\mid T_{0}, \Downarrow P_{h\rangle}}(t=0)+n_{\mid T_{0}, \Uparrow^{\left.P_{h}\right\rangle}}(t=0)=1 .
\end{aligned}
$$

The fitting parameters are $\tau_{f f}, \tau_{\text {rad }}$, and $\tau_{s}^{h}$ as well as the initial conditions which are susceptible to variations according to different applied biases used in Fig. 2. Finally, the PL dynamics detected by the APD is given by $I_{\sigma^{+} \sigma^{ \pm}}(t)=\frac{1}{\tau_{\text {rad }}} G(t) \star$ $\left[H(t) n_{\left|S_{0}, \Uparrow S_{h}\left(\Downarrow s_{h}\right)\right\rangle(t)}\right]$. When fitting simultaneously the co- and contrapolarized intensities for biases of 0.78 and $0.87 \mathrm{~V}$, we find then $\tau_{f f}=70 \mathrm{ps}, \tau_{\text {rad }}=380 \mathrm{ps}$ and $\tau_{s}^{h}=2.7 \mathrm{~ns}$. We present the deconvolution of the dynamics in Figs. 8(b) and $8(\mathrm{c})$.
[1] X. Xu, Y. Wu, B. Sun, Q. Huang, J. Cheng, D. G. Steel, A. S. Bracker, D. Gammon, C. Emary, and L. J. Sham, Phys. Rev. Lett. 99, 097401 (2007).

[2] S. Cortez, O. Krebs, S. Laurent, M. Senes, X. Marie, P. Voisin, R. Ferreira, G. Bastard, J.-M. Gérard, and T. Amand, Phys. Rev. Lett. 89, 207401 (2002).

[3] R. Oulton, A. Greilich, S. Y. Verbin, R. V. Cherbunin, T. Auer, D. R. Yakovlev, M. Bayer, I. A. Merkulov, V. Stavarache, D. Reuter, and A. D. Wieck, Phys. Rev. Lett. 98, 107401 (2007).

[4] R. V. Cherbunin, S. Y. Verbin, T. Auer, D. R. Yakovlev, D. Reuter, A. D. Wieck, I. Y. Gerlovin, I. V. Ignatiev, D. V. Vishnevsky, and M. Bayer, Phys. Rev. B 80, 035326 (2009).

[5] B. Pal, S. Y. Verbin, I. V. Ignatiev, M. Ikezawa, and Y. Masumoto, Phys. Rev. B 75, 125322 (2007).

[6] M. E. Ware, E. A. Stinaff, D. Gammon, M. F. Doty, A. S. Bracker, D. Gershoni, V. L. Korenev, S. C. Bădescu,
Y. Lyanda-Geller, and T. L. Reinecke, Phys. Rev. Lett. 95, 177403 (2005).

[7] Y. Benny, R. Presman, Y. Kodriano, E. Poem, D. Gershoni, T. A. Truong, and P. M. Petroff, Phys. Rev. B 89, 035316 (2014).

[8] P. Atkinson, E. Zallo, and O. G. Schmidt, J. Appl. Phys. 112, 054303 (2012).

[9] M. Zieliński, Y. Don, and D. Gershoni, Phys. Rev. B 91, 085403 (2015).

[10] D. Fuster, Y. González, and L. González, Nanoscale Res. Lett. 9, 309 (2014).

[11] V. Jovanov, S. Kapfinger, M. Bichler, G. Abstreiter, and J. J. Finley, Phys. Rev. B 84, 235321 (2011).

[12] The electronic resonance observed on the $X^{0}$ PLE positioned at $24 \mathrm{meV}$ could be related to the second conduction $P$-like orbital, i.e., $2 \mathrm{meV}$ above the main resonance. 
[13] E. Ivchenko and G. Pikus, Superlattices and Other Heterostructures: Symmetry and Optical Phenomena, Springer Series in Solid-State Sciences (Springer, Berlin, 1997).

[14] K. V. Kavokin, Phys. Status Solidi A 195, 592 (2003).

[15] I. V. Ignatiev, S. Y. Verbin, I. Y. Gerlovin, R. V. Cherbunin, and Y. Masumoto, Opt. Spectrosc. 106, 375 (2009).

[16] E. Poem, Y. Kodriano, C. Tradonsky, B. D. Gerardot, P. M. Petroff, and D. Gershoni, Phys. Rev. B 81, 085306 (2010).

[17] S. Laurent, M. Senes, O. Krebs, V. K. Kalevich, B. Urbaszek, X. Marie, T. Amand, and P. Voisin, Phys. Rev. B 73, 235302 (2006).

[18] K. V. Kavokin, Phys. Rev. B 69, 075302 (2004).

[19] Ş. C. Bădescu, Y. B. Lyanda-Geller, and T. L. Reinecke, Phys. Rev. B 72, 161304(R) (2005).

[20] S. Laurent, B. Eble, O. Krebs, A. Lemaître, B. Urbaszek, X. Marie, T. Amand, and P. Voisin, Phys. Rev. Lett. 94, 147401 (2005).

[21] C. Hinz, P. Gumbsheimer, C. Traum, M. Holtkemper, B. Bauer, J. Haase, S. Mahapatra, A. Frey, K. Brunner, D. E. Reiter, T. Kuhn, D. V. Seletskiy, and A. Leitenstorfer, Phys. Rev. B 97, 045302 (2018).

[22] The amplitude of the Stark shift of the excited trion is made stronger here as it depends also on the Stark shift of the hole and electron occupying $P$-like orbitals [34].

[23] M. Ediger, R. J. Warburton, K. Karrai, B. D. Gerardot, and P. M. Petroff, in Physics of Semiconductors: 27th International Conference on the Physics of Semiconductors - ICPS-27, edited by J. Menéndez and C. G. Van de Walle, AIP Conf. Proc. No. 772 (AIP, New York, 2005), p. 739.
[24] The value of $\tilde{\Delta}_{0}$ is consistent with the dark-bright exciton splitting measured by magnetospectroscopy carried out in the Voigt configuration on similar undoped samples [35].

[25] B. Urbaszek, R. J. Warburton, K. Karrai, B. D. Gerardot, P. M. Petroff, and J. M. Garcia, Phys. Rev. Lett. 90, 247403 (2003).

[26] A. B. Henriques, A. Schwan, S. Varwig, A. D. B. Maia, A. A. Quivy, D. R. Yakovlev, and M. Bayer, Phys. Rev. B 86, 115333 (2012).

[27] S. G. Carter, Ş. C. Bădescu, and A. S. Bracker, Phys. Rev. B 81, 045305 (2010).

[28] S. Germanis, P. Atkinson, R. Hostein, C. Gourdon, V. Voliotis, A. Lemaître, M. Bernard, F. Margaillan, S. Majrab, and B. Eble, Phys. Rev. B 98, 155303 (2018).

[29] A. Lafuente-Sampietro, H. Utsumi, H. Boukari, S. Kuroda, and L. Besombes, Phys. Rev. B 93, 161301(R) (2016).

[30] These terms are proportional to $\delta_{12}^{S_{e}-S_{h}}+\delta_{12}^{P_{e}-S_{h}}$.

[31] This result will be published elsewhere.

[32] Y. H. Huo, A. Rastelli, and O. G. Schmidt, Appl. Phys. Lett. 102, 152105 (2013).

[33] Considering here a fast spin relaxation time when the hole occupies the $P$ level can be regarded as an artifice reflecting the modest circular polarization of the $X^{-}$emission in Figs. 5(b) and 5(c) induced by the symmetry breaking of the QD.

[34] B. Alén, F. Bickel, K. Karrai, R. J. Warburton, and P. M. Petroff, Appl. Phys. Lett. 83, 2235 (2003).

[35] A. Pankratov, Doctoral thesis, Université Pierre et Marie CurieParis VI,2017. 